\title{
Safety and integrity management of operations in harsh environments
}

\author{
Faisal Khan ${ }^{1}$ \\ Published online: 27 May 2020 \\ (C) Springer Nature Switzerland AG 2020
}

The next industrial revolution is knocking on the door! Artificial intelligence (AI) driven automation and, more importantly, an AI-influenced workforce will dictate the direction of the "Industrial Revolution 4.0". This wonderful development will help achieve higher efficiency and more economic benefits. However, the most pertinent questions remain unanswered, "What are the safety and security implications of this development? Does this enhance safety or increase risk?" The other intriguing question is, "What are the impacts of climate change (harsh environments) on safety and integrity of operations?" The safety community must guide the current development to make it safe and sustainable.

While embracing the Industrial Revolution 4.0, explorations for natural resources are moving into harsh environments, such as Arctic and sub-Arctic regions. As a result, safety and integrity are becoming more critical for the developments in these regions. Industrial activities in harsh environments present significant technical and logistical challenges in the engineering design, construction and operation of onshore/offshore infrastructure and facilities. Due to a lack of knowledge and data, it is uncertain whether engineering specifications are sufficiently defined to ensure the safety and integrity in harsh conditions. Further complications arise as engineering schemes fall increasingly under technological, economic, environmental and human constraints. The unique features of harsh environments, including ice and permafrost, severe operating conditions, higher and more complex material degradation rates, unpredictable climatic changes, and a high degree of uncertainty, pose many different challenges that require a concerted effort from all entities involved in

Faisal Khan

fikhan@mun.ca

1 Canada Research Chair (Tier I) in Offshore Safety \& Risk Engineering, Centre for Risk Integrity and Safety Engineering (C-RISE), Faculty of Engineering \& Applied Science, Memorial University, St John's, NL A1B 3X5, Canada these endeavours. Extensive and thorough research is necessary to create new knowledge and provide the necessary understanding and information for safety and integrity engineering and management in harsh environments.

The Centre for Risk, Integrity and Safety Engineering (CRISE) at Memorial University's Faculty of Engineering and Applied Science is dedicated to developing novel techniques, investigating modern technologies and generating new knowledge. C-RISE provides an interactive platform to connect the global network of safety experts from industries, academia and R\&D organizations. The 4th Workshop and Symposium on Safety and Integrity Management of Operations in Harsh Environments (July 15-17, 2019; St John's, NL, Canada) is one such activity to exchange knowledge on safety, risk, resilience and integrity management. Through sharing the experience and knowledge, it strengthens the belief and ability to develop innovative solutions to prevent failures, incidents, vulnerability and accidents and thus improve safety, integrity and resilience.

This Special Issue includes the selective work presented and discussed at the 4th Workshop and Symposium on Safety and Integrity Management of Operations in Harsh Environments. The workshop had 6 technical sessions, 36 technical presentations, 25 keynotes, and 3 industrial training workshops. Twenty papers from the workshop were invited to submit their manuscript for this Special Issue. Submissions went through a rigorous peer-review process where nine impactful manuscripts were accepted for publication.

Maya et al. presented an interesting study on marine accidents. The study focuses on developing fuzzy cognitive maps and Bayesian networks model to learn and assess the likelihood of marine accidents. The proposed model is a novel contribution to enable a reliable assessment of potential marine accident conditions.

The engine is the heart of a ship's operation; it becomes even more critical when navigation is through arctic or subarctic conditions. Islam et al. presented a detailed emergency preparedness management plan for engineering, specific to 
harsh weather operation. This work is a practical guide to develop an effective safety management plan for Arctic and sub-Arctic operations.

Gauss et al. presented a novel approach to adapt safety assessment as part of wireless communication in a harsh environment. This work helps to develop a resilient wireless communication framework.

Icebergs present a real threat to offshore operations in many regions. One of the effective ways to manage iceberg risk is to move from its path, which demands quick disconnect and navigation capability. Fuglem et al. presented an interesting study related to the iceberg disconnect criteria for floating production systems.

Sajid et al. presented an easy and effective oil spill response model for icy conditions. The model is developed in an objectoriented Bayesian network framework. It considers the Arcticlike condition, including limited access to resources and resource capability. The proposed model will provide reliable guidance relating to oil spill response for policymakers.

Resilient design is the most effective way to design and operate in a harsher and remote location; however, there exists a limited understanding of resilience assessment and its implementation during design and operation. Towards this, Yang et al. presented a novel dynamic resilience assessment for process units operating in Arctic environments. The authors have presented an easy to implement
Bayesian model to assess and implement the resilience concept to practice.

Price et al. presented a detailed statistical comparison of different ice management conditions. The authors used simulation to generate a wide range of scenarios and relevant data. The study provides a deeper understanding of ice management strategies.

Anchors are considered ship breaks; their ability to operate reliably in the challenging condition is critical for the overall safety of the ship. Siri et al. presented a detailed study on the reliability of drag embedment anchors in the sand and the effect of idealized anchor geometry. The study provided a framework to study the range of anchor scenarios in a wide range of subsea conditions.

Talimi et al. presented a detailed numerical and experimental study of an oil spill recovery system, specifically for harsh environment conditions. The oil spill recovery method uses air sparging as a mechanism to control the oil droplet size and integrate with the suction system to collect the oil spill. The study has shown promising results.

The Editor hopes that this Special Issue will be a benefit to the scientific community and practitioners. The work presented here is a significant contribution to the knowledge base. The Editor would like to take this opportunity to congratulate the authors for their meaningful contribution to this Special Issue, and a very special "Thank You" to the reviewers for their efforts to enhance the quality of the manuscripts. 